\title{
Use of SAND Balloon Catheter for Laparoscopic Management of Extremely Large Ovarian Cysts
}

\author{
Enrico Vizza, MD, PhD, Giuseppe Cutillo, MD, PhD, Lodovico Patrizi, MD, Maria Saltari, MD, \\ Ermelinda Baiocco, $\mathrm{MD}$, and Giacomo Corrado, $\mathrm{MD}, \mathrm{PhD} *$ \\ From the Gynecologic Oncology Units, National Cancer Institute "Regina Elena," Rome (Drs. Vizza and Baiocco), National Cancer Institute "Armando \\ Businco," Cagliari (Dr. Cutillo), Department of Surgery, Section of Gynaecology and Obstetrics, "Tor Vergata” University, Rome (Drs. Patrizi and Saltari), \\ and Department of Oncology, Division of Gynaecologic Oncology, Catholic University of the Sacred Heart, Campobasso (Dr. Corrado), Italy.
}

\begin{abstract}
The objective of the study was to assess the feasibility and outcome of laparoscopic surgery for management of extremely large ovarian cysts using the SAND balloon catheter. From January 2006 to December 2009, 25 patients with large cystic adnexal tumors underwent laparoscopic-assisted surgery using the SAND balloon catheter. Median (range) patient age was 43 (20-68) years, body mass index was 25 (19-32), mass size was $26 \mathrm{~cm}(15-60 \mathrm{~cm})$, duration of the surgical procedure was 60 (30-300) minutes), and blood loss was 30 (10-100) mL. There were no major surgical complications. Intra-abdominal leakage of cyst fluid was observed in only 1 case (4\%). With proper patient selection, the SAND balloon catheter can be extremely useful in laparoscopic removal of very large adnexal masses, without intraperitoneal spillage. Journal of Minimally Invasive Gynecology (2011) 18, 779-784 (C) 2011 AAGL. All rights reserved.
\end{abstract}

Keywords: $\quad$ Adnexal mass; Laparoscopic surgery; SAND balloon catheter

DISCUSS You can discuss this case report with its authors and with other AAGL members at http://www.AAGL.org/jmig-18-5-11-00163

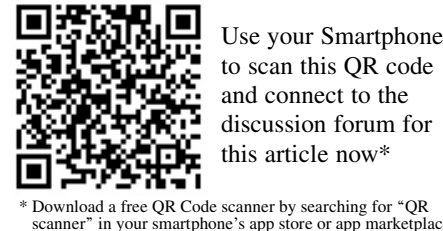

"Download a free $Q R$ Code scanner by searching for "QR
scanner" in your smartphone's app store or app marketplace.

Ovarian neoplasms are common in women of all ages. The prevalence rates of clinically detected adnexal masses in the general population is $0.17 \%$ to $5.9 \%$ in asymptomatic ovarian neoplasms, and $7.1 \%$ to $12 \%$ in symptomatic ovarian neoplasms [1]. At some time during their lifetime, at least $5 \%$ to $10 \%$ of women in the United States will undergo a surgical procedure to remove an adnexal mass [2]. During the last decade, accumulating scientific data have proved that the best approach to removal of an adnexal mass is laparoscopy [3].

Laparoscopic removal of adnexal masses has been facilitated by the use of commercially available retrieval bags. Masses as large as $18 \mathrm{~cm}$ can be removed using zipper storage bags. Masses with greater diameter have required laparotomy.

The authors have no commercial, proprietary, or financial interest in the products or companies described in this article.

Corresponding author: Giacomo Corrado, MD, PhD, Department of Oncology, Division of Gynecologic Oncology, Catholic University of the Sacred Heart, Lgo A. Gemelli 1, 86100 Campobasso, Italy.

E-mail: giacomo.corrado@alice.it

Submitted April 3, 2011. Accepted for publication June 23, 2011. Available at www.sciencedirect.com and www.jmig.org
To assess the feasibility and outcome of laparoscopic surgery for management of extremely large ovarian cysts, we studied the advantages and disadvantages of use of the SAND balloon catheter.

\section{Materials and Methods \\ Patient Characteristics}

From January 2006 to December 2009, 25 patients with clinically diagnosed large adnexal masses and low probability of malignancy were managed laparoscopically. Inclusion criteria were absence of ascites, absence of suspect sonographic and computed tomography features of the adnexal mass (absence of papillations, no suspect areas, and no reduced resistance index), no evidence of intraperitoneal spread, no enlarged pelvic lymph nodes, and only mildly elevated levels of serum tumor markers.

Preoperative evaluation of adnexal tumors was performed by examining transvaginal and transabdominal ultrasonograms to characterize the mass according to the internal echogenic pattern. Additional images were obtained using 
computed tomography or magnetic resonance imaging for further evaluation of mass characteristics, and a relevant report was made by diagnostic radiologists on the basis of standard criteria [4]. Tumor markers including CA125 and CA19.9 were measured to assist in making the diagnosis.

Each patient had an adnexal mass reaching the umbilicus or higher. All patients gave informed consent that included a statement that laparotomy might be required if cancer were detected at analysis of frozen sections.

\section{Surgical Procedure}

All operations were performed with the patient under general endotracheal anesthesia and with a nasogastric tube to the stomach. Patients were positioned in the Trendelenberg position, and the bladder was emptied using an indwelling Foley catheter. All patients were given ceftriaxone $2 \mathrm{~g}$ intravenously before surgery, and additional ceftriaxone $2 \mathrm{~g}$ was administered for the following 2 days. An intrauterine device (Uterus Manipulator; Olympus Winter \& Ibe $\mathrm{GmbH}$, Hamburg, Germany) was used in all patients.

"Open laparoscopy" was the method of entry chosen in all the cases. An incision approximately $1.5 \mathrm{~cm}$ was made around the navel, and dissection of abdominal layers was performed under vision until the peritoneal cavity was entered. In all patients, intraabdominal management was carefully standardized and included examination of the pelvis, ovaries, upper abdomen, omentum, liver, and diaphragmatic surfaces for any growths or other signs of malignancy. Then, under laparoscopic visualization, an ancillary 5-mm port was placed in the left side, $5 \mathrm{~cm}$ under the level of the umbilicus.

During the laparoscopic procedure, careful cytologic analysis of ascitic fluid, including peritoneal washing, and of cystic fluid was performed to exclude malignancy. A portion of the cystic wall was examined as a frozen section during the laparoscopic procedure, and the remainder of the cystic wall was fixed with formalin and observed under a microscope after the operation.

Tumors were punctured using the SAND balloon catheter (Hakko Shoji Co, Ltd, Tokyo, Japan), as described by Yamada et al [5] (Fig. 1). The SAND balloon catheter has an outer diameter of $2.75 \mathrm{~mm}$. In the centre of the catheter is a needle that punctures the cyst. Two balloons at the tip of the catheter grip and fix the cyst in a stationary position. The needle, with a diameter of $2.3 \mathrm{~mm}$, can be removed after the cyst is punctured, preventing tissue damage. When the 2 balloons at the tip of the catheter are filled with $10 \mathrm{~mL}$ saline solution, each swells to a diameter of approximately $25 \mathrm{~mm}$, compresses the cystic wall, and prevents spillage. The catheter was positioned through a $12-\mathrm{mm}$ trocar placed halfway between the navel and the pubic symphysis or in the right side. The cystic contents were aspired and placed in an endoscopic bag with an opening diameter of $12.7 \mathrm{~cm}$ (Endo Catch II; Tyco Healthcare Group LP, Norwalk, CT), and the cyst was extracted using the SAND balloon catheter (Fig. 2).

After the tissue was removed, the abdominal and pelvic cavities were thoroughly irrigated with copious amounts of normal saline solution. Before the procedure was terminated, any additional pelvic abnormality such as adhesions was treated, and hemostasis was secured. Ancillary accesses enlarged to more than $10 \mathrm{~mm}$ for specimen retrieval were closed in layers, whereas the umbilical incision and 5-mm ports were closed using only skin sutures.

All specimens were sent for immediate pathologic evaluation, and management decisions were made on the basis of findings in the frozen sections. In the event of malignancy, comprehensive surgical staging of apparent early-stage ovarian cancer was performed at laparoscopy. In patients with

\section{Fig. 1}

Schema of double balloon after inflation and sandwiching the cyst wall. The arrows represent the direction of the aspiration of the liquid, with the reduction of the cyst and the possibility of moving the cyst prior to removal.
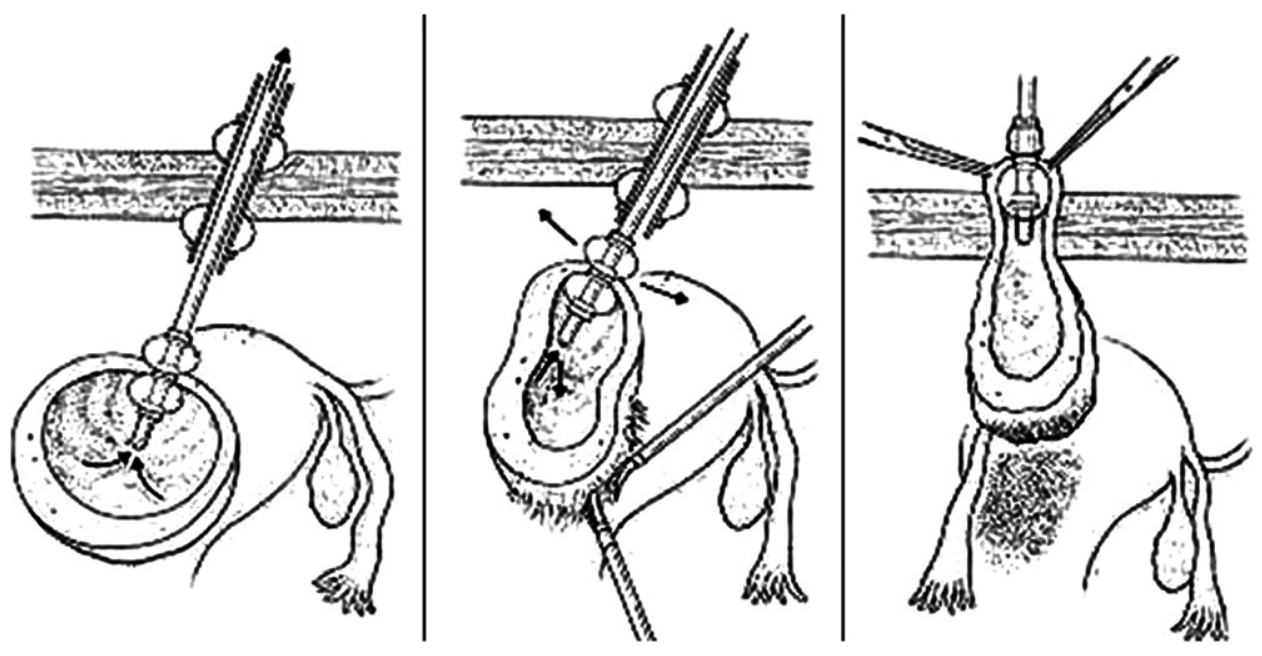
Fig. 2

A, Ovarian tumor was punctured through a side port. B and $\mathbf{C}$, Cystic contents were aspirated using the SAND balloon catheter under direct vision. D, Cyst was placed in an endobag and retrieved.
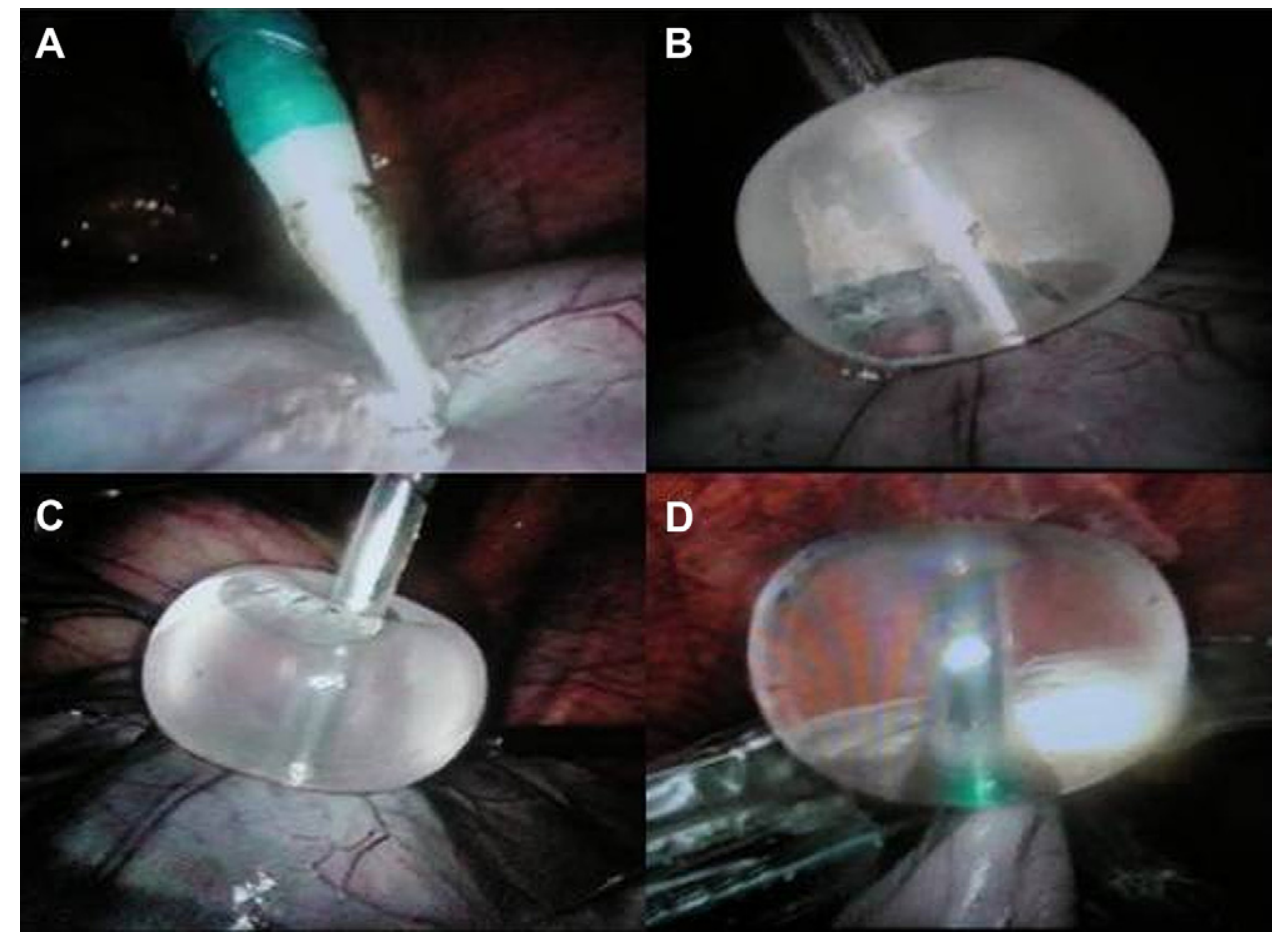

macroscopic evidence of extraovarian disease at laparoscopic examination or on the basis of findings at frozen-section analysis, cytoreductive surgery was performed at immediate laparotomy.

Cystectomy or oophorectomy were performed depending on the patient's age, obstetric history, and desire for fertility. Demographic data, indication for surgery, type of procedure, intraoperative and postoperative complications, conversions, pathologic findings, and length of stay were recorded in a prospectively maintained database. Mass size was defined as its largest diameter determined on preoperative imaging studies. Operative time was defined as the time from skin incision to skin closure. Blood loss was estimated from the volume collected in the suction device. Spillage was defined as any rupture of the tumor capsule, intentional or unintentional, potentially resulting in spilling of cyst contents into the peritoneal cavity.

\section{Results}

During the study period, 25 women met the inclusion criteria and underwent laparoscopic management of an adnexal mass $15 \mathrm{~cm}$ or larger at the National Cancer Institute "Regina Elena" in Rome, Italy. Preoperative characteristics, type of procedure, and pathologic findings are given in Table 1. Median (range) patient age was 43 (20-68) years, and body mass index was 25 (19-32). Twelve women
(48\%) were postmenopausal. Preoperative mass size was 26 (15-60) cm. Only 4 adnexal masses (16\%) were smaller than $20 \mathrm{~cm}, 12(48 \%)$ were between 20 and $30 \mathrm{~cm}$, and $9(36 \%)$ were larger than $30 \mathrm{~cm}$. The serum CA125 concentration was $77(10-300) \mathrm{U} / \mathrm{mL}$.

Surgical outcomes are given in Table 2. There were no conversions to laparotomy. No complications related to trocar placement occurred. No intraoperative or postoperative complications occurred. Median (range) fluid aspirated was 4500 (2000-10 000) mL, and spillage of benign tumor contents occurred in 1 patient (4\%). In all cases, the diagnosis at frozen-section analysis was in agreement with that at permanent section examination. A benign pathologic condition was detected in 20 of the 25 patients $(80 \%)$. Frozen-section analysis demonstrated primary ovarian cancer in 2 patients $(8 \%)$, and an ovarian tumor with low malignant potential in $3(12 \%)$. Median (range) size of benign masses was 27 (15-60) cm, of those with low malignant potential was 22 $(20-40) \mathrm{cm}$, and of malignant masses was $20(20-22) \mathrm{cm}$. No significant difference was observed in tumor size between malignant and borderline malignant tumors, whereas benign tumors were larger than malignant masses. Median mass size was larger in premenopausal women than in postmenopausal women, at $29.5(15-60) \mathrm{cm}$ vs $20(18-21) \mathrm{cm}$.

Frozen-section analysis demonstrated that 2 patients had ovarian cancer. Both cancers were staged entirely at laparoscopy. There was no evidence of extraovarian disease at 


\begin{tabular}{|c|c|}
\hline \multicolumn{2}{|l|}{ Table 1} \\
\hline \multicolumn{2}{|c|}{$\begin{array}{l}\text { Clinical characteristics in } 25 \text { women with large ovarian cysts treated } \\
\text { at laparoscopic surgery using the SAND balloon catheter }\end{array}$} \\
\hline Variable & Value \\
\hline \multicolumn{2}{|l|}{ Demographic data, mean (range) } \\
\hline Age, yr & $43(20-68)$ \\
\hline Body mass index & $25(19-32)$ \\
\hline Preoperative mass size, $\mathrm{cm}$ & $77(10-300)$ \\
\hline \multicolumn{2}{|l|}{ Type of surgery, No. (\%) } \\
\hline Cystectomy & $11(44)$ \\
\hline Unilateral salpingo-oophorectomy & $2(8)$ \\
\hline Bilateral salpingo-oophorectomy & $7(28)$ \\
\hline $\begin{array}{l}\text { Total abdominal hysterectomy and } \\
\text { bilateral salpingo-oophorectomy }\end{array}$ & $3(12)$ \\
\hline $\begin{array}{l}\text { Total laparoscopic hysterectomy, bilateral } \\
\text { salpingo-oophorectomy, and } \\
\text { cytoreductive surgery }\end{array}$ & $1(4)$ \\
\hline $\begin{array}{l}\text { Total abdominal hysterectomy, bilateral } \\
\text { salpingo-oophorectomy, and } \\
\text { cytoreductive surgery }\end{array}$ & $1(4)$ \\
\hline \multicolumn{2}{|l|}{ Histologic findings, No. (\%) } \\
\hline Endometriotic cyst & $1(4)$ \\
\hline Cystic teratoma & $1(4)$ \\
\hline Serous cystoadenoma & $10(40)$ \\
\hline Mucinous cystoadenoma & $8(32)$ \\
\hline $\begin{array}{l}\text { Ovarian tumor with low malignant } \\
\text { potential }\end{array}$ & $3(12)$ \\
\hline Malignant epithelial tumor & $2(8)$ \\
\hline
\end{tabular}

laparoscopic examination, and the final histologic analysis demonstrated a serous ovarian carcinoma, FIGO stage IA, moderately differentiated. Both patients received adjuvant chemotherapy with a combination of carboplatin and paclitaxel. After follow-up of 60 and 48 months, respectively, both patients are alive with no evidence of disease.

All tumors with low malignant potential were managed laparoscopically, and 1 of 3 patients (33.3\%) underwent

\begin{tabular}{lc|}
\hline Table 2 & \\
\hline $\begin{array}{l}\text { Surgical outcome in patients with large ovarian cysts treated at } \\
\text { laparoscopic surgery using the SAND balloon catheter }\end{array}$ \\
& Value \\
Variable & $60(30-300)$ \\
Operative time, min & $30(10-100)$ \\
Blood loss, mL & $4500(2000-10000)$ \\
Volume of fluid aspirated, mL & $1(4)$ \\
Spillage, No. of patients $(\%)$ & 0 \\
Intraoperative or postoperative complications & 0 \\
Blood transfusion & 0 \\
Conversion to other surgical technique & 0 \\
Repeat intervention & $3(2-7)$ \\
Hospital stay, day & \\
\hline a Median values. & \\
\hline
\end{tabular}

fertility-sparing procedures. After median (range) followup of 35 (25-47) months, all women with a diagnosis of tumor with low malignant potential are alive with no evidence of disease. Length of hospital stay in the entire study group was 3 (2-7) days.

\section{Discussion}

Findings of the present study suggest that use of the SAND balloon catheter in laparoscopic surgery to treat ovarian cysts is feasible and beneficial in a large percentage of women with a large adnexal mass if performed appropriately and by experienced surgeons.

Management of the patient with a large adnexal mass has traditionally required laparotomy with a vertical midline incision to enable adequate access and to avert intraoperative cyst rupture. When compared with laparoscopic procedures, the open approach often results in longer hospitalization, increased intraoperative blood loss, greater postoperative pain, more analgesic requirements, and longer outpatient recovery time before return to work or usual daily activities [6]. The literature reports many reasons for restricting the laparoscopic approach to treatment of large adnexal masses. The risk of encountering a malignant ovarian tumor is the most frequently cited reason in adnexal masses smaller than 10 $\mathrm{cm}$. Although the size of an adnexal mass is an independent predictor of the risk of its being malignant [7], many, if not most, large ovarian tumors are benign. In a review of 1648 histopathologically diagnosed ovarian masses, benign disease was detected in $68 \%$ of cysts between 10 and $19 \mathrm{~cm}$ in greatest diameter, and in $48 \%$ of cysts with a diameter of $20 \mathrm{~cm}$ or more [8]. Moreover, no randomized trials have been published to date that compare the laparoscopic surgical staging of presumed early ovarian cancer using conventional open staging. A number of cohort studies have attested to the feasibility and safety of the laparoscopic approach to ovarian cancer staging, and they are consistent in demonstrating low rates of conversion to open procedures and reasonable intraoperative complication rates. Tumors treated laparoscopically consistently were associated with less intraoperative blood loss and either shorter postoperative hospitalization or quicker bowel function recovery than with open surgery [9].

In the present study, 2 (8\%) separate cases of ovarian cancer were diagnosed on the basis of results of frozen-section analysis of an intact ovarian mass. Both tumors were staged entirely at laparoscopy because no evidence of extraovarian disease had been demonstrated. No trocar metastasis or tumor dissemination was observed, and after follow-up of 60 months, the patient is alive without disease.

Diagnosis by frozen-section analysis was in agreement with that of permanent section in all of our cases. Evidence from several studies suggests that specificity of frozensection analysis is consistently high in all sizes of adnexal tumours, whereas sensitivity is particularly reduced in large tumors, especially those with mucinous histologic 
features [10]. Under these circumstances, a laparoscopic approach to primary surgery in women with a large mass later proved malignant may decrease the likelihood of subjecting women with understaged lesions to repeat laparotomy, which can ultimately result in significantly higher morbidity. The availability of specialized pathologists at our institution and analysis of multiple sections in large masses could have contributed to the high accuracy of frozen-section diagnosis in our study.

The high rate of capsular rupture is another reason not to perform laparoscopy in the setting of large adnexal masses. The reported incidence of spillage of tumor contents in published series of laparoscopically managed large adnexal masses varies between $22 \%$ and $100 \%$, probably reflecting differences in the definition of spillage rather than surgical technique [11]. No prospective comparative data support the existence of increased tumor spillage in women with ovarian cancer managed using laparoscopic techniques, and unequivocal evidence that cyst rupture in early ovarian cancer worsens the prognosis is lacking. A recent randomized study of laparoscopy vs laparoscopy-guided minilaparotomy for management of large adnexal masses reported a relative risk of cyst rupture of 5.5 in women undergoing laparoscopy compared with minilaparotomy [12]. However, the mean diameter of the masses was approximately $8 \mathrm{~cm}$ in both groups, and cysts larger than $18 \mathrm{~cm}$ were excluded. Thus, it remains to be determined whether these results can be held true for larger masses that invariably require decompression for removal.

Some authors report that spontaneous dehiscence of the cyst wall can occur after the first puncture, spilling the cyst contents into the abdominal cavity before the balloons are applied [13]. Therefore, the SAND balloon needle is not applicable to cysts with thin walls. This concept is certainly valid. However, a Medline search of English and non-English literature published between 2000 and 2010 [5,14-22], conducted to identify other reports of laparoscopic surgery using the SAND balloon catheter to treat cysts, yielded 10 studies, accounting for a total of 93 patients with a median (range) preoperative mass size of $8.5(4.2-12.5 \mathrm{~cm})$, with no reported spillage (Table 3 ). In the present study, tumor spillage was low, occurring in only 1 patient (4\%) due to damage to the cyst wall through a small incision. The patient had a polycystic mucinous cystoadenoma $45 \mathrm{~cm}$ in greatest diameter with $5500 \mathrm{~mL}$ of highly viscous material inside. After extensive washing of the abdominal cavity with saline solution, the peritoneal fluid was normal.

We believe that tumor spillage is a serious concern but is not of sufficient justification to recommend open surgery in all women with a large ovarian cyst, provided the intent of gynecologic cancer surgery is not sacrificed for the benefits of laparoscopy. With the SAND balloon catheter, it is possible to aspirate the cystic fluid with virtually no spillage. The cystic fluid in the cavity of the cyst is removed via repeated injection of warm physiologic saline solution and suctioning. However, because minimal spillage may not be completely prevented, even with careful use of the SAND balloon catheter, laparoscopic monitoring is important for assessment of the degree of accidental spillage of tumor contents. After completion of the surgical procedure, extensive washing of the entire abdominal and pelvic cavities under laparoscopic guidance should be performed to flush out any possible spillage of tumor contents, especially when the possibility of spillage is suspected in mucinous cystic tumors, which are most frequently encountered in the treatment of large adnexal tumors.

In conclusion, although our experience is not extensive, we believe that the SAND double-balloon catheter is a useful, easy, and safe method for aspiration of large ovarian cyst contents and also for their laparoscopic resection. Moreover, the present study adds to accumulating evidence that supports the safety and excellent surgical outcome of laparoscopic surgery performed to treat large benign ovarian cysts.

\begin{tabular}{|c|c|c|c|c|c|c|c|c|}
\hline \multicolumn{9}{|c|}{ Reports of laparoscopic surgery to treat cysts using the SAND balloon catheter } \\
\hline Yamada et al [5], 2000 & 32 & 31 & 6.3 & NA & 114 & NA & NA & $0 / 32$ \\
\hline Shimokawa et al [15], 2001 & 2 & NA & 4.25 & NA & NA & NA & NA & $0 / 2$ \\
\hline Yamada et al [16], 2001 & 1 & 48 & 10 & 35 & NA & NA & 250 & $0 / 1$ \\
\hline Yamada et al [17], 2002 & 1 & 87 & NA & 5458.2 & 95 & NA & 5000 & $0 / 1$ \\
\hline Takeda et al [18], 2006 & 1 & 9 & 12 & 17.3 & 77 & 50 & NA & $0 / 1$ \\
\hline Ito et al [19], 2006 & 1 & 34 & 7 & NA & NA & NA & NA & $0 / 1$ \\
\hline Takeda et al [20], 2007 & 39 & 51.5 & NA & 21.9 & 62 & 30 & 1857 & $0 / 39$ \\
\hline
\end{tabular}




\section{Supplementary Data}

Supplementary data related to this article can be found online at doi:10.1016/j.jmig.2011.06.019.

\section{References}

1. Padilla LA, Radosevich DM, Milad MP. Accuracy of the pelvic examination in detecting adnexal masses. Obstet Gynecol. 2000;96:593-598.

2. NIH Consensus Development Panel on Ovarian Cancer. NIH consensus conference. Ovarian cancer: screening, treatment, and follow-up. JAMA. 1995;273:491-497.

3. Canis M, Rabischong B, Houlle C, et al. Laparoscopic management of adnexal masses: a gold standard? Curr Opin Obstet Gynecol. 2002;14: 423-428.

4. Jung SE, Lee JM, Rha SE, Byun JY, Jung JI, Hahn ST. CT and MR imaging of ovarian tumors with emphasis on differential diagnosis. Radiographics. 2002;22:1305-1325.

5. Yamada T, Okamoto Y, Kasamatsu H. Use of the SAND balloon catheter for the laparoscopic surgery of benign ovarian cysts. Gynaecol Endosc. 2000;9:51-54.

6. Sagiv R, Golan A, Glezerman M. Laparoscopic management of extremely large ovarian cysts. Obstet Gynecol. 2005;105:1319-1322.

7. Timmerman D, Testa AC, Bourne T, et al. International Ovarian Tumor Analysis Group. Logistic regression model to distinguish between the benign and malignant adnexal mass before surgery: a multicenter study by the International Ovarian Tumor Analysis Group. J Clin Oncol. 2005;23:8794-8801.

8. Okugawa K, Hirakawa T, Fukushima K, Kamura T, Amada S, Nakano H. Relationship between age, histological type, and size of ovarian tumors. Int J Gynaecol Obstet. 2001;74:45-50.

9. Ghezzi F, Cromi A, Siesto G, Serati M, Zaffaroni E, Bolis P. Laparoscopy staging of early ovarian cancer: our experience and review of the literature. Int J Gynaecol Cancer. 2009;19(Suppl 2):S7-S13.

10. Fanfani F, Zannoni GF, Fagotti A, et al. Importance of a specialized pathologist for the examination of frozen sections of adnexal masses. Int $J$ Gynaecol Cancer. 2007;17:1034-1039.
11. Eltabbakh GH, Charboneau AM, Eltabbakh NG. Laparoscopic surgery for large benign ovarian cysts. Gynecol Oncol. 2008;108:72-76.

12. Benedetti Panici P, Palaia I, Bellati F, Pernice M, Angioli R, Muzii L. Laparoscopy compared with laparoscopically guided minilaparotomy for large adnexal masses: a randomized controlled trial. Obstet Gynecol. 2007;110:241-248.

13. Shozu M, Segawa T, Sumitani H, Inoue M. Leak-proof puncture of ovarian cysts: instant mounting of plastic bag using cyanoacrylate adhesive. Obstet Gynecol. 2001;97:1007-1010.

14. Yamada T, Okamoto Y, Kasamatsu H, Mori H. Laparoscopic-assisted surgery for benign ovarian cyst in a young girl. J Am Assoc Gynecol Laparosc. 2001;8:295-298.

15. Shimokawa S, Watanabe S, Sakasegawa K, Nakamura Y, Hisashi Y, Sakata R. Balloon catheter for cyst aspiration in a thoracoscopic resection of mediastinal cysts. Surg Today. 2001;31:284-286.

16. Yamada T, Okamoto Y, Kasamatsu H, Mori H. Laparoscopic-assisted removal of a large urachal cyst. J Am Assoc Gynecol Laparosc. 2001; 8:159-160.

17. Yamada T, Kasamatsu H, Mori H. Laparoscopic-assisted surgery and irradiation in an 87-year-old woman with a large ovarian cystic tumour and uterine cervical cancer. Gynaecol Endosc. 2002;11:431-433.

18. Takeda A, Manabe S, Mitsui T, Nakamura H. Laparoscopic management of mature cystic teratoma of bilateral ovaries with adnexal torsion occurring in a 9-year-old premenarchal girl. J Pediatr Adolesc Gynecol. 2006; 19:403-406.

19. Ito M, Harada T, Yamauchi N, Tsudo T, Mizuta M, Terakawa N. Small bowel perforation from a thermal burn caused by contact with the end of a laparoscope during ovarian cystectomy. J Obstet Gynaecol Res. 2006; 32:434-436.

20. Takeda A, Sakai K, Mitsui T, Nakamura H. Management of large cystic adnexal tumor by gasless laparoscopic-assisted surgery with wound retractor. J Minim Invasive Gynecol. 2007;14:644-650.

21. Takeda A, Imoto S, Mori M, Sakai K, Nakamura H. Laparoscopic management of mature cystic teratoma of the ovary with multiple intracystic floating spherules. Arch Gynecol Obstet. 2009;279:775-776.

22. Takeda A, Imoto S, Mori M, Nakano T, Nakamura H. Wound retraction system for isobaric laparoendoscopic single-site surgery to treat adnexal tumors: pilot study. J Minim Invasive Gynecol. 2010;17:626-630. 